\title{
Inhibitory effect of Embelin on human acute $T$ cell lymphoma Jurkat cells through activation of the apoptotic pathway
}

\author{
XIU-LI ZHU ${ }^{1}$, LIAN JIANG ${ }^{1}$, FAN QU $^{1}$, ZHI-YU WANG ${ }^{2}$ and LIAN-MEI ZHAO ${ }^{3}$ \\ Departments of ${ }^{1}$ Pediatrics, ${ }^{2}$ Immunology and Immunotherapy, ${ }^{3}$ Research Center, \\ Fourth Hospital of Hebei Medical University, Shijiazhuang, Hebei 050011, P.R. China
}

Received September 28, 2014; Accepted May 14, 2015

DOI: $10.3892 / \mathrm{ol} .2015 .3364$

\begin{abstract}
It has previously been shown that Embelin inhibits proliferation, promotes apoptosis, and increases sensitivity and reduces resistance to chemotherapy drugs, in various types of tumor cells. The present study examined the effects of Embelin on the proliferation of human acute $\mathrm{T}$ cell lymphoma Jurkat cells. Jurkat cells were treated with various concentrations of Embelin and the effects of Embelin on the inhibition of growth of Jurkat cells were evaluated. Expression of X-linked inhibitor of apoptosis protein (XIAP); poly ADP ribose polymerase; caspase-3; caspase-8; caspase-9; the proapoptotic protein, Bax; and the antiapoptotic proteins, $\mathrm{Bcl}-\mathrm{xl}$ and Bcl-2, were assessed. The results showed that Embelin significantly inhibited the growth of human acute T cell lymphoma Jurkat cells. Following treatment with 5, 10 or $20 \mathrm{mM}$ Embelin for $48 \mathrm{~h}$, cell viability was $82.31,58.65$ and $37.62 \%$, respectively, which was significantly reduced compared with that of the control group and the $0.1 \%$ DMSO control group $(\mathrm{P}<0.01)$. Furthermore, the caspase-3 inhibitor, z-DEVD-fmk, and the caspase-9 inhibitor, Ac-LEHD-CHO, reversed this inhibitory effect. It was also shown that the apoptotic rate of cells treated with Embelin was significantly elevated. Subsequently, it was demonstrated that Embelin downregulated the expression of XIAP and the proapoptotic Bcl2 family members, Bcl-2 and Bcl-xl, while it concomitantly upregulated that of the antiapoptotic protein, Bax. These results showed that Embelin inhibited growth and induced apoptosis of Jurkat cells in vitro, by activating the endogenous caspase-dependent apoptotic pathway through inhibition of XIAP and proapoptotic Bcl-2 family members.
\end{abstract}

Correspondence to: Dr Lian-Mei Zhao, Research Center, Fourth Hospital of Hebei Medical University, 12 Jiankang Road, Shijiazhuang, Hebei 050011, P.R. China

E-mail: lianmeizhmail@163.com

Key words: Embelin, lymphoma, Jurkat, apoptosis, X-linked inhibitor of apoptosis protein

\section{Introduction}

Lymphoma is one of the most common types of malignant tumors in children and the incidence of this disease is increasing (1). Non-Hodgkin's lymphoma accounts for $~ 80-85 \%$ of the total number of lymphoma cases, and exhibits a high degree of malignancy, rapid progression and a poor prognosis in children (2). A novel strategy for the treatment of lymphoma, is the induction of apoptosis of lymphoma cells. However, the majority of currently available apoptosis inducers contain large quantities of heavy metals and thus produce a number of intolerable side effects $(3,4)$. Therefore, the identification of novel inducers of apoptosis in lymphoma cells, which exhibit higher efficiency and lower toxicity, is required.

Embelin is a small molecule inhibitor of X-linked inhibitor of apoptosis (XIAP), which is used in traditional Chinese medicine (5-7). Previous studies have shown that Embelin inhibits proliferation, promotes apoptosis, increases sensitivity to chemotherapeutic agents and reduces drug resistance in tumor cells (8-12). Therefore, Embelin has been identified as a promising antitumor agent. However, to date there have been few studies conducted, examining the effect of Embelin on acute $\mathrm{T}$ cell lymphoma. The present study investigated the inhibitory effect of Embelin on human acute T cell lymphoma Jurkat cells, in addition to the mechanisms underlying this effect.

\section{Materials and methods}

Cell culture. Human acute $\mathrm{T}$ cell lymphoma Jurkat cells (Chinese Academy of Medical Sciences, Beijing, China) were cultured in RPMI 1640 medium (Applied Biosystems China Ltd., Beijing, China), containing $100 \mathrm{U} / \mathrm{ml}$ penicillin and $100 \mu \mathrm{g} / \mathrm{ml}$ streptomycin (Life Technologies, Grand Island, NY, USA) at $37^{\circ} \mathrm{C}$ with $5 \% \mathrm{CO}_{2}$. Cells were treated with $0.25 \%$ trypsin (Applied Biosystems China Ltd.,) for passage, and cells in the logarithmic growth phase were selected for subsequent experiments. Embelin was purchased from Sigma-Aldrich (St. Louis, MO, USA). Caspase inhibitors, z-DEVD-fmk and Ac-LEHD-CHO, were obtained from Promega Corporation (Madison, WI, USA).

MTS/PMS method. The MTS/PMS method was used to evaluate the inhibition rate of tumor cell proliferation. Jurkat cells were divided into three groups that were treated with various 
concentrations of Embelin (5, 10 or $20 \mathrm{mM}$; Hebei Medical University, Haisen Pharmaceutical Co., Ltd., Hebei, China). The control group was incubated with an equal quantity of RPMI 1640 medium. RPMI 1640 medium with no cells was used as a blank control group, and the cell viability of each sample was measured 3 times, and the mean of the 3 values was calculated. Jurkat cells in the logarithmic growth phase were cultured in RPMI 1640 medium containing 10\% fetal bovine serum (Hangzhou Sijiqing Biological Engineering Materials Co., Ltd., Zhejiang, China ). The cell concentration was adjusted to $1 \times 10^{5} / \mathrm{ml}$, and cells were inoculated into a 96-well culture plate; each well containing $90 \mu 1$ of culture medium. Plates were incubated at $37^{\circ} \mathrm{C}$ with $5 \%$ $\mathrm{CO}_{2}$. Following incubation for $24 \mathrm{~h}, 10 \mu \mathrm{l}$ Embelin at various concentrations $(5,10$ or $20 \mathrm{mmol} / \mathrm{ml})$ was added to the appropriate wells. Culture medium containing $0.01 \%$ dimethyl sulfoxide (DMSO; Tianjin Yongda Chemical Reagents Development Center, Tianjin, China) was added to the control group for the MTS assay.

In subsequent experiments, the caspase- 3 inhibitor, z-DEVD-fmk, and the caspase-9 inhibitor, Ac-LEHD-CHO, were added to cells, to achieve a final concentration of $10 \mu \mathrm{l}$. The different concentrations of Embelin were added $1 \mathrm{~h}$ later, and cells were then cultured for $24 \mathrm{~h}$. The MTS/PMS mixture (20 $\mu \mathrm{l}$ ) was added to the cells, and plates were cultured for a further 3-4 h. Morphological changes were observed under an inverted microscope (Olympus Corporation, Tokyo, Japan) under 40X magnification. A fully automated enzyme mark measuring instrument (Titertek Multiskan, North Ryde, Australia) was used to detect the absorbance of the samples at $570 \mathrm{~nm}$ wavelength. Cell viability was calculated as follows: Viability $(\%)=[$ experimental group (OD) - blank group (OD)]/[control group (OD) - blank group (OD)] x 100.

Phosphatidylserine ectropion [Annexin V/propidium iodide (PI)] analysis. Phosphatidylserine ectropion (Annexin V/PI) analysis were used to analyze rates of cell apoptosis. Culture medium $(2 \mathrm{ml})$ with various concentrations of Embelin (5, 10 or $20 \mathrm{mM}$ ) were added to Jurkat cells in the logarithmic growth phase as the drug treatment groups. Complete culture medium was used for the control group. After $24 \mathrm{~h}$, the cells were collected, centrifuged at $850 \times g$ for $5 \mathrm{~min}$ and then washed three times with phosphate buffer solution. Centrifugation $(5 \mathrm{~min})$ was performed following each wash. Cells were resuspended in $2 \mathrm{ml}$ of phosphate buffer solution for $5 \mathrm{~min}$. Subsequently, $5 \mu \mathrm{l}$ Annexin V-FITC (Lianke Biological Engineering Co., Ltd., Zhejiang, China) and $10 \mu \mathrm{l}$ propidium iodide (Lianke Biological Engineering Co., Ltd.) were added, and samples were incubated for $10 \mathrm{~min}$ at room temperature. Cells were stained in darkness at $4^{\circ} \mathrm{C}$ for $30 \mathrm{~min}$, and flow cytometry (Beckman Coulter, Inc., USA) was then used to analyze the rate of apoptosis. In the results of the apoptosis analysis, the left upper, right upper, left lower and right lower quadrants represent necrotic cells, late apoptotic cells, normal cells and early apoptotic cells, respectively. The right lower quadrant was selected for evaluation of the levels of apoptosis in Jurkat cells.

Western blotting. Western blotting was performed as described previously (13). Briefly, cells treated with 5,10 , or $20 \mathrm{mM}$ Embelin for $24 \mathrm{~h}$ were lysed, and protein concentrations

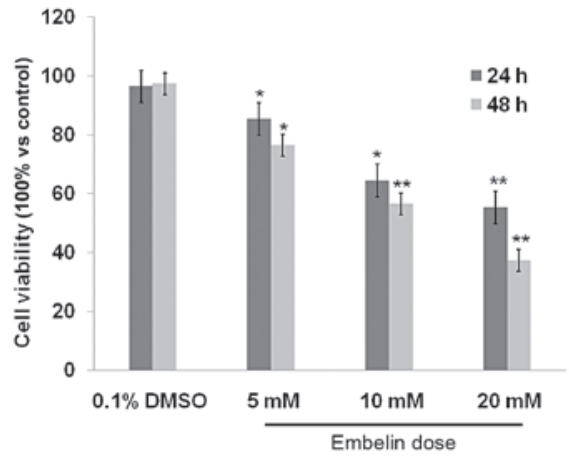

Figure 1. Effect of Embelin on the proliferation of Jurkat cells. Viability represents the percentage of viable Jurkat cells measured using an MTS assay. ${ }^{*} \mathrm{P}<0.05$, vs. control group.

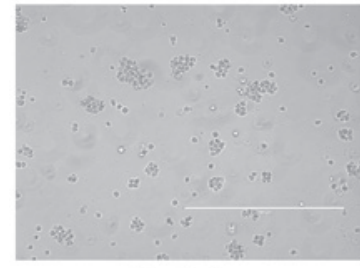

Control

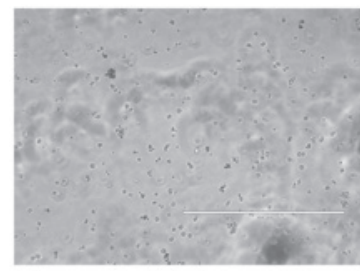

$10 \mathrm{mM}$ Embelin

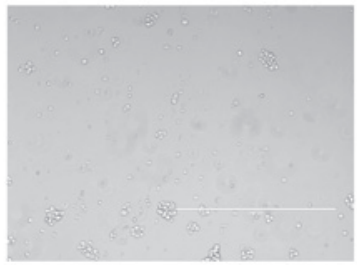

$5 \mathrm{mM}$ Embelin

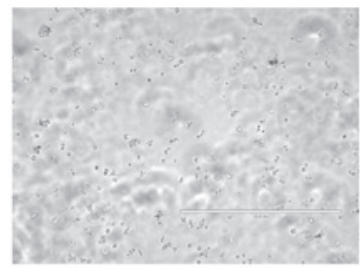

$20 \mathrm{mM}$ Embelin
Figure 2. Effect of Embelin on the proliferation and cell morphology of Jurkat cells. Representative images were captured. Magnification, x200.

were determined using a Lowry protein assay kit (Sigma Corporation of America, New York, NY, USA). Protein samples $(50 \mu \mathrm{g})$ were separated using SDS-PAGE (Millipore Corporation, Billerica, MA, USA), transferred to a PVDF membrane, blocked in $5 \%$ dried non-fat milk for $1 \mathrm{~h}$ at $25^{\circ} \mathrm{C}$, and then incubated with primary rabbit polyclonal antibodies to XIAP, (cat. no, sc-11426; 1:1,000), and Caspase 3, 8 and 9 (cat. no. sc-7148; 1:500), and mouse monoclonal antibody to PARP (cat. no. sc-56196; 1:1,000) were purchased from Santa Cruz Biotechnology, Inc.(Dallas, TX, USA) and primary mouse monoclonal antibody to GADPH (cat. .no. 2118S; $1: 10,000)$ and primary rabbit polyclonal antibodies to $\mathrm{Bcl}-2$ (cat. no. 2870P; 1:1,000), Bcl-XL (cat. no. 2764P; 1:1,000) and Bax (cat. .no. 2774S, 1:1,000) were purchased from Cell Signaling Technology, Inc. (CST, CA, USA) overnight at $4^{\circ} \mathrm{C}$. Membranes were washed 3 times for 5 min with TBST and incubated for $1 \mathrm{~h}$ with fluorochrome-labeled secondary antibodies against rabbit or mouse (1:10,000; IRDye $800-\mathrm{LI}-\mathrm{COR}$ for rabbit antibodies or IRDye 700-LI-COR for mouse antibodies; LI-COR Biosciences, Ltd., Cambridge, UK). Following 4 washes with TBST, expression of the target proteins were analyzed by imaging the membrane with a LI-COR Odyssey infrared imager (LI-COR Biosciences, Ltd.). GAPDH was used as an internal control. 
Table I. Percentage of apoptotic Jurkat cells following treatment with various doses of Embelin.

\begin{tabular}{lcrr}
\hline Group & Healthy cells $(\%)$ & Apoptotic cells $(\%)$ & Dead cells $(\%)$ \\
\hline Control (DMSO) & $95.94 \pm 0.35$ & $3.05 \pm 0.73$ & $1.01 \pm 0.04$ \\
Embelin $5 \mathrm{mmol} / \mathrm{ml}$ & $87.35 \pm 0.94$ & $9.65 \pm 1.20^{\mathrm{a}}$ & $2.31 \pm 0.61$ \\
Embelin $10 \mathrm{mmol} / \mathrm{ml}$ & $58.24 \pm 1.53$ & $32.45 \pm 3.08^{\mathrm{a}}$ & $12.45 \pm 2.04$ \\
Embelin $20 \mathrm{mmol} / \mathrm{ml}$ & $50.16 \pm 0.69$ & $40.79 \pm 2.78^{\mathrm{a}}$ & $13.64 \pm 2.21$
\end{tabular}

${ }^{\mathrm{a}} \mathrm{P}<0.05$, vs. the control group.

Statistical analysis. Data are reported as the mean \pm standard error of the mean. A one-way analysis of variance (ANOVA) was performed to determine the significance between groups. Tukey's method was used for multiple comparisons. $\mathrm{P}<0.05$ was considered to indicate a statistically significant difference. SPSS software, version 13.0 (SPSS, Inc., Chicago, IL, USA) was used for data analysis.

\section{Results}

Effect of Embelin on the proliferation of Jurkat cells. The results of the MTS assay showed that all concentrations of Embelin used in the present study, significantly inhibited the proliferation of Jurkat $\mathrm{T}$ cell lymphoma cells in a dose- and time-dependent manner, compared with proliferation of cells in the control group. As shown in Fig. 1, following treatment with 5, 10 and $20 \mathrm{mM}$ for $48 \mathrm{~h}$, cell viability was $\sim 82.31,58.65$ and $37.62 \%$ respectively, which was significantly reduced compared with that in the control group and the $0.1 \%$ DMSO control group $(\mathrm{P}<0.01)$. It was also shown that the number of viable cells visible under the microscope was significantly reduced (Fig. 2). These results demonstrated the potency of Embelin in inhibiting the growth of $\mathrm{T}$ cell lymphoma cancer cells in vitro.

Effect of Embelin on the rate of apoptosis in Jurkat cells. Induction of apoptosis is the primary mechanism of action of Embelin. Therefore the mechanism underlying the induction of cell death by Embelin was examined using flow cytometry. The results showed that all concentrations of Embelin used, significantly increased the rates of apoptosis of Jurkat $\mathrm{T}$ cell lymphoma cells, compared with rates in the control group $(\mathrm{P}<0.05)$. The percentage of apoptotic cells following treatment with 5, 10 and $20 \mathrm{mM}$ Embelin, was $~ 9.65,32.45$ and $40.79 \%$ respectively. An association between higher drug concentrations and increased apoptosis was also observed $(\mathrm{P}<0.05$; Fig. 3, Table I). These data suggest that Embelin induces apoptosis of Jurkat cells in a dose-dependent manner.

Effect of Embelin on activity of the caspase pathway in Jurkat cells. In order to examine the molecular pathways underlying the effects of Embelin on Jurkat cells, western blot analysis was performed to measure the activity of apoptosis-related, caspase proteins. The results showed that treatment of Jurkat cells with Embelin for $48 \mathrm{~h}$ resulted in cleavage of caspase-3, as demonstrated by the appearance of a $19 \mathrm{kDa}$ intermediate. Furthermore, treatment of Jurkat
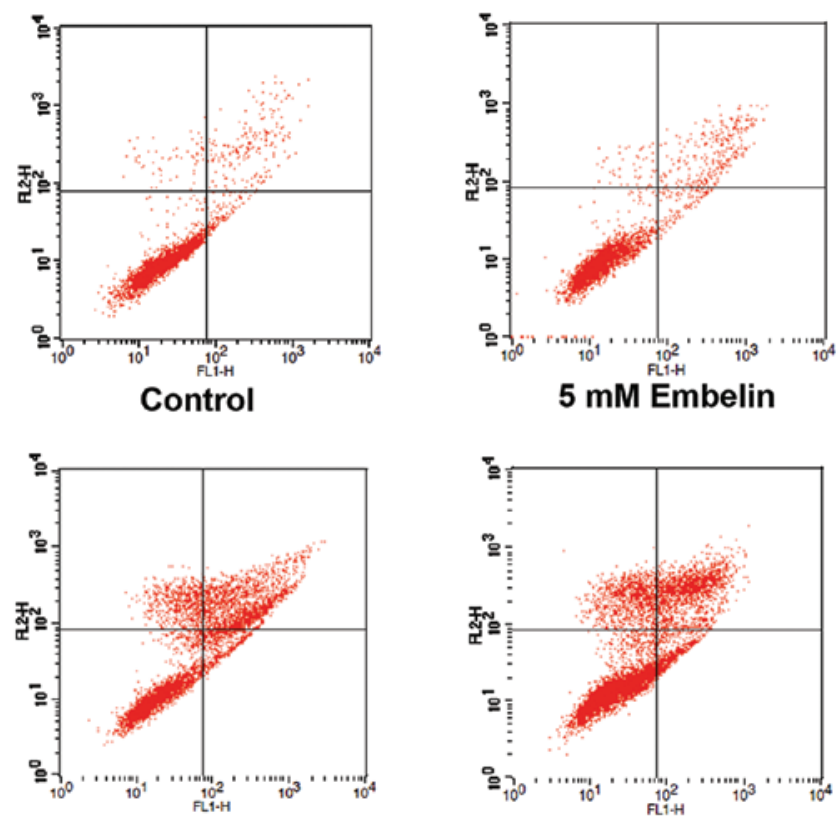

$10 \mathrm{mM}$ Embelin

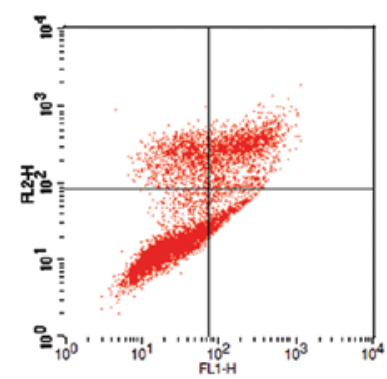

20 mM Embelin

Figure 3. Effect of Embelin on the rate of apoptosis rate of Jurkat cells. Left upper, right upper, left lower and right lower quadrants represent necrotic cells, late apoptotic cells, healthy cells and early apoptotic cells, respectively. The right lower quadrant was selected for evaluation of the level of apoptosis in Jurkat cells.

cells with Embelin also resulted in significantly increased levels of cleaved caspase-9, with no concomitant change in the levels of procaspase- 8 (Fig. 4A and B). Based on these results, it was hypothesized that Embelin triggers Jurkat cell apoptosis through the intrinsic but not the extrinsic pathway. Notably, treatment with the caspase-3 inhibitor, z-DEVD-fmk, and the caspase-9 inhibitor, Ac-LEHD-CHO, reduced the inhibitory effects of Embelin on Jurkat $\mathrm{T}$ cell lymphoma cells $(\mathrm{P}<0.05$; Fig. 4C). Activation of caspases during apoptosis partly accounts for the cleavage of certain cellular substrates, such as poly ADP-ribose polymerase (PARP). Therefore PARP has been adopted as a visual marker of caspase-3 activity in the intrinsic apoptosis pathway. As shown in Fig. 4, the levels of cleaved PARP fragment (the active form) were significantly increased following the exposure of cells to Embelin for $48 \mathrm{~h}$, further clarifying the activity of caspase-3 in Jurkat cells. These results also suggested that Embelin induces apoptosis of Jurkat cells via the intrinsic apoptotic pathway. 
A
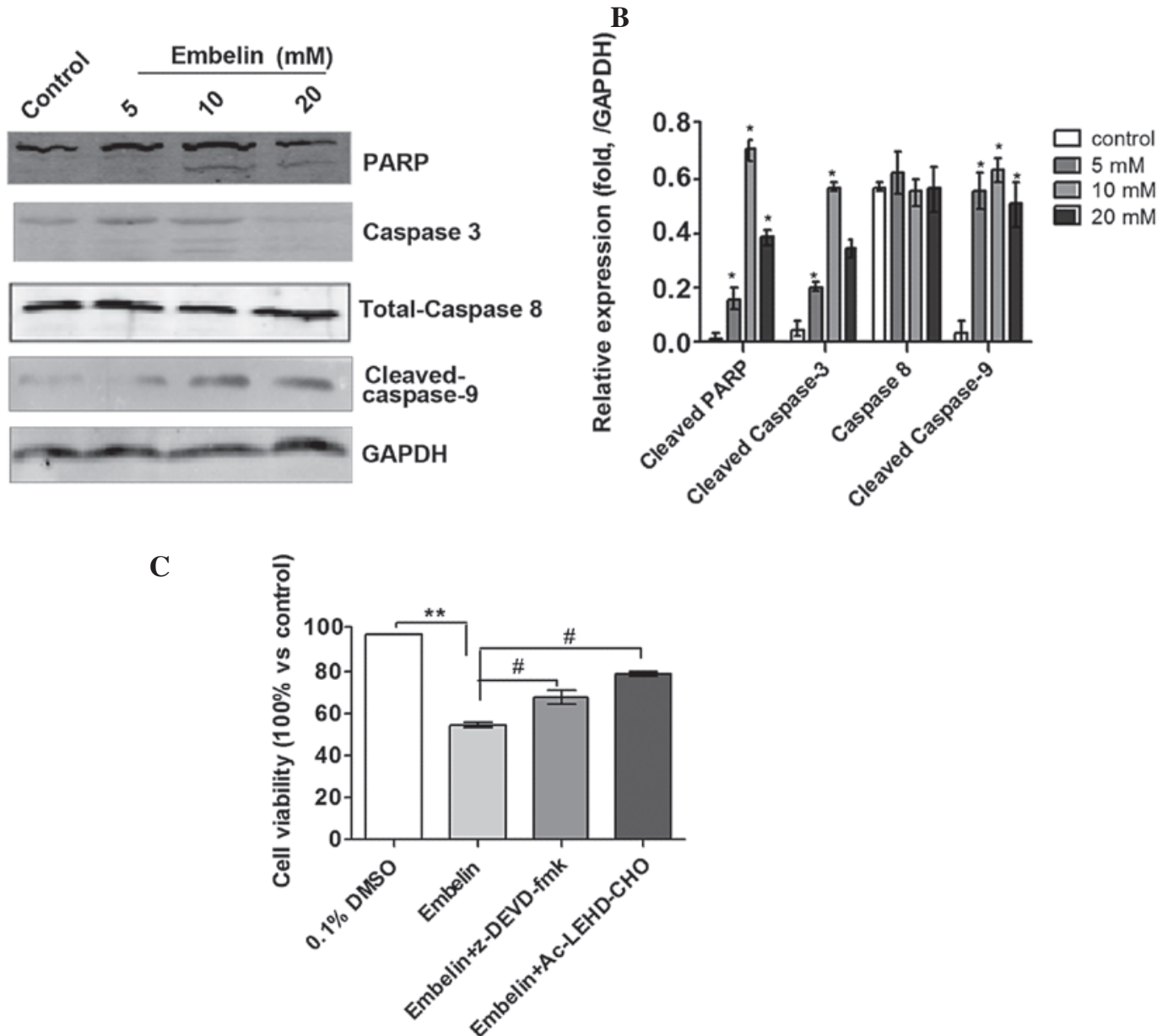

Figure 4. Effect of Embelin on the activity of the caspase pathway and effect of the caspase inhibitors, z-DEVD-fmk and Ac-LEHD-CHO, on Embelin-treated Jurkat cells. (A) Effect of Embelin on the expression of caspase 3, 8 and 9, and PARP. Experiments were repeated in triplicate. (B) Quantitative analysis of the results presented in (A). (C) Effect of Embelin combined with caspase inhibitors, z-DEVD-fmk and Ac-LEHD-CHO, on the viability of Jurkat cells. ** P $<0.01$, vs. $0.1 \%$ DMSO control group and ${ }^{\#} \mathrm{P}<0.05$, vs. $20 \mathrm{mM}$ Embelin treatment group. PARP, poly ADP ribose polymerase; DMSO, dimethyl sulfoxide.
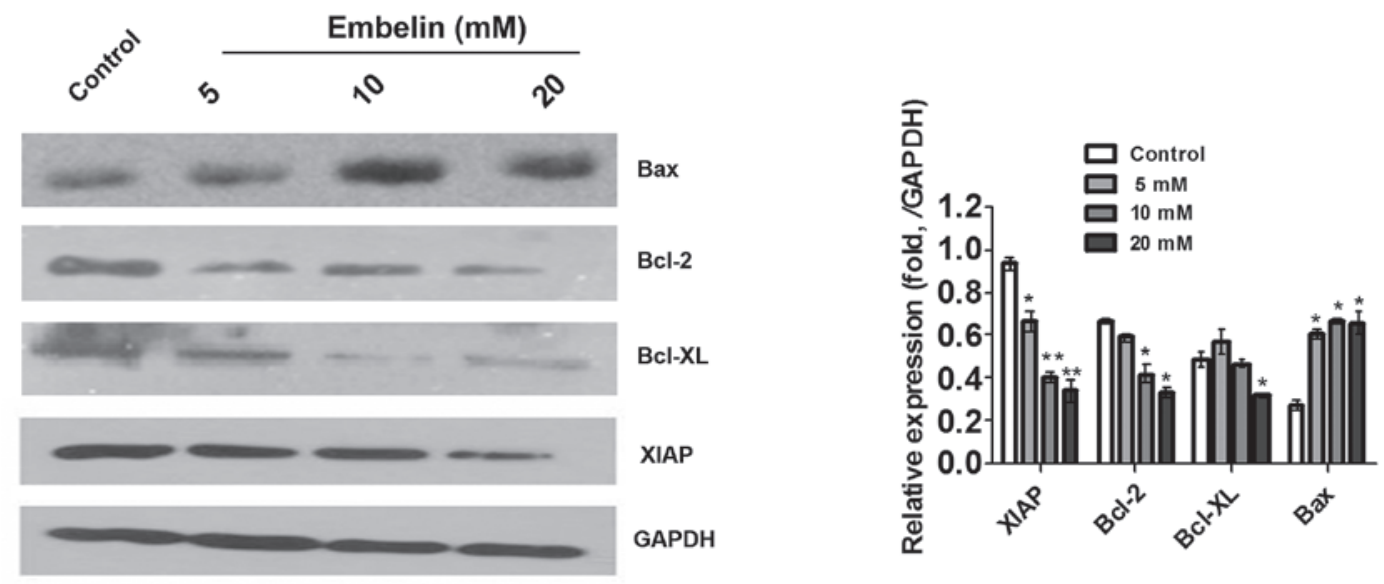

Figure 5. Effect of Embelin on the expression of XIAP and Bcl-2 family proteins in Jurkat cells. " $\mathrm{P}<0.05$, vs. the control group. XIAP, X-linked inhibitor of apoptosis protein.

Effect of Embelin on the expression of apoptosis-related proteins in Jurkat cells. Embelin is a small molecule inhibitor of XIAP that is used in traditional Chinese medicine. The results of the present study showed that 5-20 mM Embelin downregulated the expression of XIAP in Jurkat cells (Fig. 5), suggesting that Embelin induces apoptosis of Jurkat cells, 
in part through regulation of the expression of XIAP. Mitochondrial integrity is regulated by the Bcl-2 family, which is composed of proapoptotic members, including Bcl-2 and Bcl-xl, and antiapoptotic members, such as Bax $(14,15)$. Therefore, in the present study, the expression of these Bcl-2 family members was detected in the Jurkat cells, following treatment with various concentrations of Embelin for $48 \mathrm{~h}$. As shown in Fig. 5, decreased expression of Bcl-2 and Bcl-xl accompanied by an increased expression of Bax was observed in Jurkat cells. These results demonstrated that regulation of the Bcl-2 family also is involved in the induction of apoptosis in Jurkat cells by Embelin.

\section{Discussion}

XIAP is a recently discovered key member of the IAP family and is the strongest inhibitor of apoptosis among the IAP proteins (16). XIAP functions to inhibit the activity of caspases, and therefore regulates apoptosis via multiple pathways (17). Overexpression of the XIAP gene is detected in the majority of tumor cell lines and is closely associated with the progression, recurrence, prognosis and resistance to chemotherapy of tumor cells (18). XIAP overexpression has also been demonstrated in a number of types of highly malignant tumor cell lines and is associated with resistance to chemotherapy of tumor cells (19). Embelin is a small molecule inhibitor of XIAP, used in traditional Chinese medicine that binds the Smac binding site in the BIR3 domain of XIAP, thus blocking the inhibitory effects of XIAP on caspases, increasing the level of active caspases, and thereby promoting apoptosis $(20,21)$.

The present study examined the effects of Embelin on human acute $\mathrm{T}$ cell lymphoma Jurkat cells. Embelin inhibited the proliferation of acute lymphoma Jurkat cells in a dose-dependent manner. Furthermore, the caspase-3 inhibitor, z-DEVD-fmk, and the caspase-9 inhibitor, Ac-LEHD-CHO, inhibited the induction of apoptosis by Embelin. Western blotting results demonstrated that XIAP protein expression in the Embelin treatment groups was significantly decreased, compared with that in the control group $(\mathrm{P}<0.05)$. In addition, it was shown that Embelin treatment led to the activation of caspase-3 and PARP. Furthermore, as the concentration of Embelin increased, the levels of caspase-3 and PARP cleavage fragments also increased. Caspase- 3 is a member of the caspase family, and functions as the executor of apoptosis; its activation thus being important for cell apoptosis. Activation of caspase-3 is primarily responsible for the cleavage of PARP and ultimately results in cell apoptosis $(22,23)$.

The current study also confirmed that Embelin induces apoptosis by inhibiting the expression of XIAP and activating the caspase pathway. In addition, it was shown that following Embelin treatment, there was a dose-dependent increase in the level of the caspase- 9 cleavage fragment in the Embelin treatment groups (5 and $10 \mathrm{mM}$ Embelin) compared with the control group, however this effect did not increase further with $20 \mathrm{mM}$ Embelin treatment, while no significant changes in the level of caspase- 8 were detected. The present study also demonstrated that Embelin upregulated the proapoptotic protein, Bax, and downregulated the expression of the antiapoptotic proteins, Bcl-2 and Bcl-xl, indicating that Embelin induces apoptosis via activation of the mitochondrial pathway.
These results suggested that Embelin may induce apoptosis of Jurkat cells by inhibiting XIAP, Bcl-2 and Bcl-xl, and by increasing the expression of Bax, thus activating the caspase cascade reaction.

In conclusion, the current study showed that Embelin inhibits the growth and induces apoptosis of Jurkat cells in vitro, and that these effects are associated with its effect on XIAP and the intrinsic apoptotic pathway. These results suggest that Embelin may be a candidate for the treatment of T cell lymphoma.

\section{Acknowledgements}

This study was supported by the Natural Science Foundation of China (grant no. 81173611) and the Major Infrastructure Projects of Hebei Science and Technology (grant no. 2011011) and was organized by The Fourth Hospital of Hebei Medical University, China.

\section{References}

1. Andrade AF, Borges KS, Castro-Gamero AM, Silveira VS, Suazo VK, Oliveira JC, Moreno DA, de Paula Queiroz RG, Scrideli CA and Tone LG: Zebularine induces chemosensitization to methotrexate and efficiently decreases AhR gene methylation in childhood acute lymphoblastic leukemia cells. Anticancer Drugs 25: 72-81, 2014.

2. Stiff PJ, Unger JM, Cook JR, Constine LS, Couban S, Stewart DA, Shea TC, Porcu P, Winter JN, Kahl BS, et al: Autologous transplantation as consolidation for aggressive non-Hodgkin's lymphoma. N Engl J Med 369: 1681-1690, 2013.

3. Plosker GL and Figgitt DP: A review of its use in non-Hodgkin's lymphoma and chronic lymphocytic leukaemia. Drugs 63: 803-843, 2003.

4. Till KJ, Coupland SE and Pettitt AR: Motility and trafficking in B-cell non-Hodgkin's lymphoma (Review). Int J Oncol 45: 5-12, 2014.

5. Moreno-Martínez D, Nomdedeu M, Lara-Castillo MC, Etxabe A, Pratcorona M, Tesi N, Díaz-Beyá M, Rozman M, Montserrat E, Urbano-Ispizua A, et al: XIAP inhibitors induce differentiation and impair clonogenic capacity of acute myeloid leukemia stem cells. Oncotarget 12: 4337-4346, 2014.

6. Wang A, Zhang B, Zhang J, Wu W and Wu W: Embelin-induced brain glioma cell apoptosis and cell cycle arrest via the mitochondrial pathway. Oncol Rep 29: 2473-2478, 2013.

7. Dai Y, Jiao H, Teng G, Wang W, Zhang R, Wang Y, Hebbard L, George J and Qiao L: Embelin reduces colitis-associated tumorigenesis through limiting IL-6/STAT3 signaling. Mol Cancer Ther 13: 1206-1216, 2014.

8. Yao GH, Luan JF, Ye D, Yan JM, Lei QH, Zhu PY and Jin J: Effects of triptolide on proliferation and apoptosis of Jurkat cell line in acute T lymphocytic leukemia. Zhongguo Shi Yan Xue Ye Xue Za Zhi 16: 506-509, 2008 (In Chinese).

9. Hu R, Wu B, Zhang GJ, Wang HT, Zhu K, Yang W and Liu ZG: Effect of Embelin on proliferation, differentiation and apoptosis of HL-60 cells. Zhonghua Xue Ye Xue Za Zhi 31: 442-445, 2010 (In Chinese).

10. Marsh JL, Jackman CP, Tang SN, Shankar S and Srivastava RK: Embelin suppresses pancreatic cancer growth by modulating tumor immune microenvironment. Front Biosci (Landmark Ed) 19: 113-125, 2014.

11. Cheng YJ1, Jiang HS, Hsu SL, Lin LC, Wu CL, Ghanta VK and Hsueh CM: XIAP-mediated protection of H460 lung cancer cells against cisplatin: Eur J Pharmacol 627: 75-84, 2010.

12. Deveraux QL, Takahashi R, Salvesen GS and Reed JC: X-linked IAP is a direct inhibitor of cell-death proteases. Nature 388: 300-304, 1997.

13. Zhao L, Shan B, Du Y, Wang M, Liu L and Ren FZ: Periplocin from Cortex periplocae inhibits cell growth and down-regulates survivin and c-myc expression in colon cancer in vitro and in vivo via beta-catenin/TCF signaling. Oncol Rep 24: 375-383, 2010.

14. Danial NN: Bcl-2 family proteins: Critical checkpoints of apoptotic cell death. Clin Cancer Res 13: 7254-7263, 2007. 
15. Silke J and Vucic D. IAP family of cell death and signaling regulators: Methods Enzymol 545: 35-65, 2014.

16. Kuwana T and Newmeyer DD: Bcl-2-family proteins and the role of mitochondria in apoptosis. Curr Opin Cell Biol 15: 691-699, 2003.

17. Bao W, Zhu F, Duan Y, Yang Y and Cai H. HtrA1 resensitizes multidrug-resistant hepatocellular carcinoma cells by targeting XIAP. Biomed Pharmacother 70: 97-102, 2015.

18. Chen FH, Qu HY and Sui GJ: Effects of apoptosis protein XIAP inhibito on the apoptosis and sensitivity of chemotherapy in A549 cell. Chin J Lung Cancer 11: 368-372, 2008 (In Chinese).

19. Nikolovska-Coleska Z, Xu L, Hu Z, et al: Discovery of embelin as a cell-permeable, small-molecular weight inhibitor of XIAP through structure-based computational screening of a traditional herbal medicine three-dimensional structure database. J Med Chem 47: 2430-2440, 2004.
20. Irshad S, Ashworth A and Tutt A: Therapeutic potential of PARP inhibitors for metastatic breast cancer. Expert Rev Anticancer Ther 11: 1243-1251, 2011.

21. Nikrad M, Johnson T, Puthalalath H, Coultas L, Adams J and Kraft AS: The proteasome inhibitor bortezomib sensitizes cells to killing by death receptor ligand TRAIL via BH3-only proteins Bik and Bim. Mol Cancer Ther 4: 443-449, 2005.

22. Wong RS: Apoptosis in cancer: From pathogenesis to treatment. J Exp Clin Cancer Res 30: 87, 2011.

23. Ulukaya E, Acilan C and Yilmaz Y: Apoptosis: Why and how does it occur in biology. Cell Biochem Funct 29: 468-480, 2011. 